\title{
Treatment with Sulforaphane Produces Antinociception and Improves Morphine Effects during Inflammatory Pain in Mice
}

\author{
Alejandro Redondo, Pablo Aníbal Ferreira Chamorro, Gabriela Riego, Sergi Leánez, \\ and Olga Pol
}

Grup de Neurofarmacologia Molecular, Institut d'Investigació Biomèdica Sant Pau \& Institut de Neurociències, Universitat Autònoma de Barcelona, Barcelona, Spain

Received August 9, 2017; accepted September 19, 2017

\begin{abstract}
The activation of nuclear factor erythroid 2-related factor 2 (Nrf2) exerts potent antioxidative and anti-inflammatory effects; however, its participation in the modulation of chronic inflammatory pain and on the antinociceptive effects of $\mu$-opioid receptor (MOR) agonists has not been evaluated. We investigated whether the induction of Nrf2 could alleviate chronic inflammatory pain and augment the analgesic effects of morphine and mechanisms implicated. In male C57BL/6 mice with inflammatory pain induced by complete Freund's adjuvant (CFA) subplantarly administered, we assessed: 1) antinociceptive actions of the administration of 5 and $10 \mathrm{mg} / \mathrm{kg}$ of a Nrf2 activator, sulforaphane (SFN); and 2) effects of SFN on the antinociceptive actions of morphine and on protein levels of Nrf2, heme oxygenase $1(\mathrm{HO}-1)$, and $\mathrm{NAD}(\mathrm{P}) \mathrm{H}$ : quinone oxidoreductase 1 (NQO1) enzymes, microglial activation and inducible nitric
\end{abstract}

oxide synthase (NOS2) overexpression, as well as on mitogenactivated protein kinase (MAPK) and MOR expression in the spinal cord and paw of animals with inflammatory pain. Results showed that treatment with SFN inhibited allodynia and hyperalgesia induced by CFA and increased the local antinociceptive actions of morphine. This treatment also augmented the expression of Nrf2, HO-1, NQO1, and MOR, and inhibited NOS2 and CD11b/c overexpression and MAPK phosphorylation induced by inflammation. Thus, this study shows that the induction of Nrf2 might inhibit inflammatory pain and enhance the analgesic effects of morphine by inhibiting oxidative stress and inflammatory responses induced by peripheral inflammation. This study suggests the administration of SFN alone and in combination with morphine are potential new ways of treating chronic inflammatory pain.

\section{Introduction}

Chronic inflammatory pain is a recurrent disorder, affecting approximately at $20 \%$ of the world's population. The actual therapies (nonsteroidal anti-inflammatory drugs, opioids, etc.) may be limited in scope, have dose-limiting side effects, which reflects the need to search for new strategies to treat more effectively this type of pain.

The transcription factor nuclear factor erythroid 2-related factor 2 (Nrf2) regulates two important cytoprotective pathways: antioxidative and anti-inflammatory (Kim et al., 2010). A crucial factor for the antioxidant activity of Nrf2 is the regulation of the antioxidant response element found in the promoter regions of numerous genes that encode antioxidant and detoxification enzymes, for example, heme oxygenase 1(HO-1), $\mathrm{NAD}(\mathrm{P}) \mathrm{H}$ quinone oxidoreductase 1 (NQO1), superoxide dismutase 1, etc., which maintain the homeostatic equilibrium

This work was supported by the Ministerio de Economía y Competitividad, Instituto de Salud Carlos III, Madrid, Spain, and Fondo Europeo de Desarrollo Regional, Unión Europea [Grants PS0900968 and PI1400927].

P.A.F.C. is a recipient of a BECAL fellowship from the Paraguay Consejo Nacional de Ciencia y Tecnología, Ministerio de Educación y Ciencias, and Ministerio de Hacienda.

https://doi.org/10.1124/jpet.117.244376. by regulating the reactive oxygen species cumulating. Recent investigations have also demonstrated that Nrf2 activation, in addition to modulating oxidative stress, is also able to directly inhibit the transcription of cytokines [interleukin (IL)- $1 \beta$, IL-6] by an independent mechanism of antioxidant response element and reactive oxygen species levels (Kobayashi et al., 2016). Therefore, while the lack of Nrf2 augmented the inflammatory response in the joints and the expression of the tumor necrosis factor- $\alpha$ and IL-6 accelerating the arthritis signs (Maicas et al., 2011), its activation reduced inflammation by decreasing the synthesis of several proinflammatory mediators, such as the inducible isoform of nitric oxide synthase (NOS2), cyclooxygenase-2, IL-1 $\beta$, IL-6, and the mitogen-activated protein kinase (MAPK) in the cartilage from osteoarthritic animals (Kim et al., 2009; Davidson et al., 2013). More interesting is the result that the activation of Nrf2 also reduced acute pain provoked by nitroglycerin and neuropathic pain associated with diabetes or generated by nerve injury in rodents (Negi et al., 2011; Di et al., 2016; McDonnell et al., 2017; Wang and Wang, 2017). Nevertheless, the role played by Nrf2 on the allodynia and hyperalgesia induced by chronic peripheral inflammation has not been studied.

ABBREVIATIONS: ANOVA, analysis of variance; CFA, complete Freund's adjuvant; ERK, extracellular signal regulated kinase; HO-1, heme oxygenase 1; IL, interleukin; JNK, C-Jun N-terminal kinase; MAPK, mitogen-activated protein kinase; MOR, $\mu$-opioid receptor; NOS2, inducible nitric oxide synthase; NQO1, NAD(P)H: quinone oxidoreductase 1; Nrf2, nuclear factor erythroid 2-related factor 2; SFN, sulforaphane. 
We and other authors have demonstrated that treatment with HO-1 inducer compounds, such as the cobalt protoporphyrin IX, not only inhibits chronic pain but also potentiates the antinociceptive properties of $\mu$-opioid receptor (MOR) agonists during neuropathic and inflammatory pain (Hervera et al., 2013a,b; Carcolé et al., 2014). Based on these results, we postulated that Nrf2 induction could also play an important role in the modulation of the antinociceptive effects of morphine (a MOR agonist) in animals with chronic inflammatory pain.

It is well known that activated microglia is implicated in the progress of inflammatory and neuropathic pain (Martini et al., 2016) by mediating the synthesis of abundant inflammatory mediators (tumor necrosis factor- $\alpha$, IL, NOS2, etc.) and its inhibition reduced allodynia and hyperalgesia in animals with chronic pain (Pol, 2007; Hervera et al., 2012; Mika et al., 2013). In addition, MAPK also exercises a crucial role in the regulation of inflammatory reactions. That is, during inflammatory pain c-Jun N-terminal kinase (JNK), extracellular signal regulated kinase (ERK) 1/2, and p38 MAPK are differentially activated in neurons and glial cells and its inhibition attenuates pain (Gao and Ji, 2008; Ji et al., 2009). Nevertheless, the role played by sulforaphane (SFN), a Nrf2 inducer compound, in the modulation of these inflammatory mediators in mice with chronic inflammatory pain is still unknown.

In complete Freund's adjuvant (CFA)-induced chronic inflammatory pain in mice we assessed: 1) the antiallodynic and antihyperalgesic effects of the repetitive intraperitoneal administration of 5 and $10 \mathrm{mg} / \mathrm{kg}$ of SFN; 2) the antinociceptive effects of the administration of $\mathrm{SFN}$ in combination with a subanalgesic dose of morphine; and 3) the effects of SFN on the expression of Nrf2, HO-1, NQO1, NOS2, CD11b/c (microglial marker), MOR, and MAPK (JNK, ERK1/2, and p38) activation in the spinal cord and paw of CFA-injected mice.

\section{Materials and Methods}

Experimental Animals. For experimental procedures, male C57BL/6 mice acquired from Envigo (Barcelona, Spain) were employed. The weight of all mice was between 21 and $25 \mathrm{~g}$. Mice were accommodated under 12-hour/12-hour light/dark conditions in a room with controlled temperature of $22^{\circ} \mathrm{C}$ and humidity of $66 \%$. Animals with free access to food and water were used after a minimum of 7 days acclimatization to the housing conditions. All experiments were executed according to the Guide for the Care and Use of Laboratory Animals as adopted and promulgated by the National Institutes of Health (Bethesda, MD) and approved by the local Committee of Animal Use and Care of the Autonomous University of Barcelona. Maximal exertions to reduce animal suffering and number of animals employed were made.

Induction of Chronic Inflammatory Pain. We induced chronic inflammatory pain via subplantar injection of CFA (30 $\mu \mathrm{l}$; SigmaAldrich, St. Louis, MO) into the right hindpaw under brief anesthetic conditions with isoflurane according to our previous studies (Hervera et al., 2009; Leánez et al., 2009). Mechanical allodynia was measured by means of von Frey filaments and thermal hyperalgesia was measured by the plantar test. Animals were checked in both behavioral tests before and at 3, 4, 7, 10, and 14 days after CFA injection.

Nociceptive Behavioral Tests. Mechanical allodynia was measured by evaluating the hindpaw withdrawal response to von Frey filament stimulation. Mice were sited in methacrylate cylinders (20 cm high $\times 9 \mathrm{~cm}$ in diameter; Servei Estació, Barcelona, Spain) on a wire grid bottom across which von Frey filaments (North Coast Medical, Inc., San Jose, CA) with bending force in the range of 0.008-3.5 $\mathrm{g}$ were applied by employing an adapted version of the up/down paradigm described by Chaplan et al. (1994). We start the test with a filament of $0.4 \mathrm{~g}$, and a filament of $3.0 \mathrm{~g}$ was used as the cutoff. The strength of the next filament was increased or decreased in accordance with the response. The threshold of response was calculated from the sequence of filament strength used during the up/down procedure using an Excel program (Microsoft Iberia SRL, Barcelona, Spain), which includes curve fitting of the data. A clear paw withdrawal, licking the paw, or shaking the paw was considered to be a nociceptivelike response. Both ipsilateral and contralateral hindpaws were tested.

Thermal hyperalgesia was evaluated by evaluating paw withdrawal latency in response to radiant heat by using the plantar test apparatus (Ugo Basile, Varese, Italy) and in accordance with the method proposed by Hargreaves et al. (1988). Mice were placed in methacrylate cylinders (20 cm high $\times 9 \mathrm{~cm}$ in diameter) situated on a glass surface. The heat source was positioned under the plantar surface of both hindpaws (contralateral and ipsilateral) and activated with a light beam intensity selected in previous works with baseline latencies from 8 to 10 seconds under control conditions. To avoid tissue damage in lack of response, a cutoff time of 12 seconds was established. The mean paw withdrawal latencies were calculated from the average of three distinct trials, taken at 5-minute pauses to avoid thermal sensitization and behavioral disturbances. In all of these nociceptive behavioral assays, mice were habituated to the test for 1 hour before testing to obtain suitable behavioral immobility, and they were performed by a male experimenter blinded to the treatments applied.

Western Blot Analysis. Animals were sacrificed by cervical dislocation at 0 (naive) and 14 days after CFA injection from SFNor vehicle-treated mice. Tissues from the ipsilateral side of the lumbar section of the spinal cord or hindpaw were extracted quickly after killing, frozen in liquid nitrogen, and maintained at $80^{\circ} \mathrm{C}$ until use. For all tissues, samples from four animals were pooled together to have adequate levels of proteins for Western blot assays. The protein levels of Nrf2, HO-1, NQO1, NOS2, CD11/bc, MOR, and MAPK (pJNK/JNK, pERK1/2/ERK1/2, and pp38/p38) were analyzed. The homogenization of tissues was performed in icecold lysis buffer (50 mM Tris B Base, $150 \mathrm{nM} \mathrm{NaCl}, 1 \% \mathrm{NP}-40,2 \mathrm{mM}$ EDTA, $1 \mathrm{mM}$ phenylmethylsulfonyl fluoride, 0.5 Triton X-100, 0.1\% sodium dodecyl sulfate, $1 \mathrm{mM} \mathrm{Na} \mathrm{VO}_{4}, 25 \mathrm{mM} \mathrm{NaF}, 0.5 \%$ protease inhibitor cocktail, and 1\% phosphatase inhibitor cocktail). Except for NP-40, which was purchased from Calbiochem (Darmstadt, Germany), all other reagents were acquired from Sigma-Aldrich. After solubilization of crude homogenate for 1 hour at $4^{\circ} \mathrm{C}$, crude homogenate was sonicated for 10 seconds and centrifuged at $4^{\circ} \mathrm{C}$ for 15 minutes at $700 \mathrm{~g}$. Then, the supernatant (60 $\mu \mathrm{g}$ of total protein) was mixed with $4 \times$ laemmli loading buffer and loaded onto $4 \%$ stacking/10\% separating sodium dodecyl sulfate polyacrylamide gels. After that, proteins were electrophoretically transferred onto a polyvinylidene fluoride membrane for 120 minutes and then blocked with phosphate-buffered saline plus $5 \%$ nonfat dry milk or Tris-buffered saline with Tween 20 plus $5 \%$ nonfat dry milk or $5 \%$ bovine serum albumin for 1 hour and 15 minutes, and then incubated with specific rabbit primary antibody anti-Nrf2 (1:160; Abcam, Cambridge, United Kingdom); HO-1 (1:300; Abcam), NQO1 (1:333; Sigma-Aldrich), NOS2 (1:200; Abcam), CD11b/c (1:200; Novus Biologic, Littleton, CO), MOR (1:333; Merck, Billerica, MA), phospho-JNK, total JNK, phospho-ERK1/2, total ERK1/2, and total p38 (1:250; Cell Signaling Technology, Danvers, MA), and phospho-p38 (1:200; Cell Signaling Technology) overnight at $4^{\circ} \mathrm{C}$. A horseradish peroxidase-conjugated anti-rabbit secondary antibody (GE Healthcare, Little Chalfont, United Kingdom) was used to detect proteins, which were visualized with chemiluminescence reagents (ECL kit; GE Healthcare) and by exposure onto hyperfilm (GE Healthcare). Blot intensity was quantified by densitometry. Stripped membranes were reproved with a rabbit anti-glyceraldehyde-3-phosphate dehydrogenase antibody (1:5000; Merck) used as a loading control.

Experimental Procedure. Baseline responses were established after the habituation period, beginning with the von Frey filaments 
A
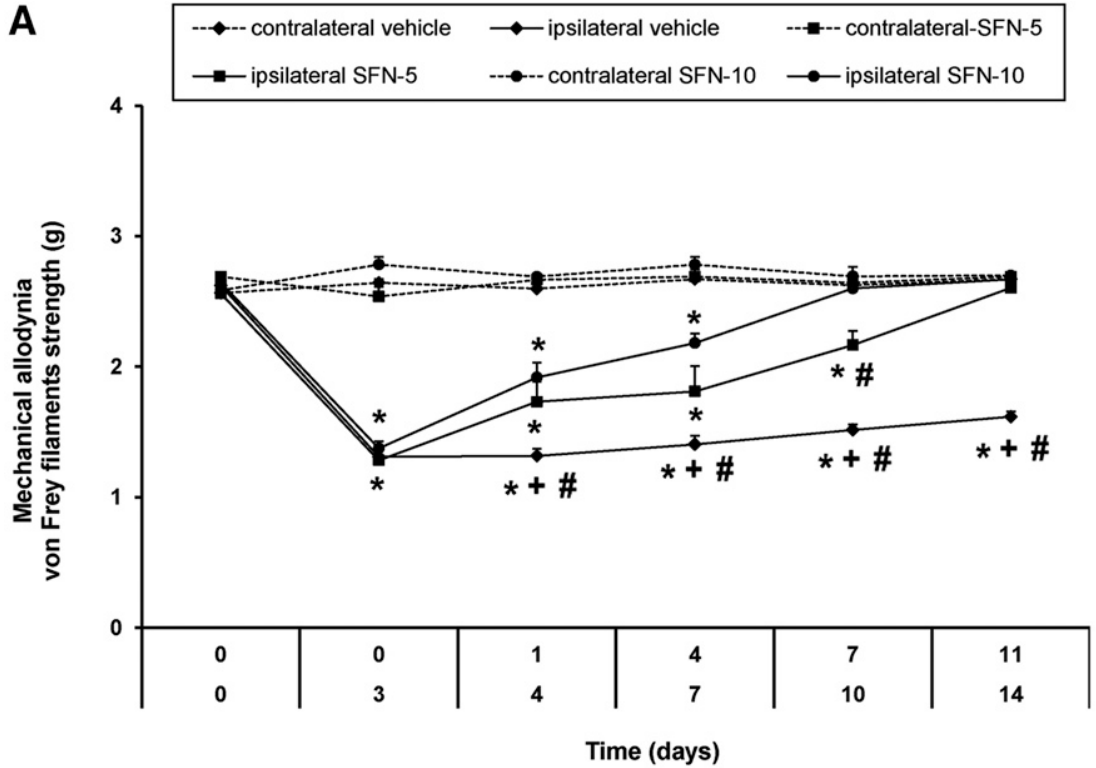

B

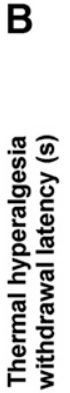

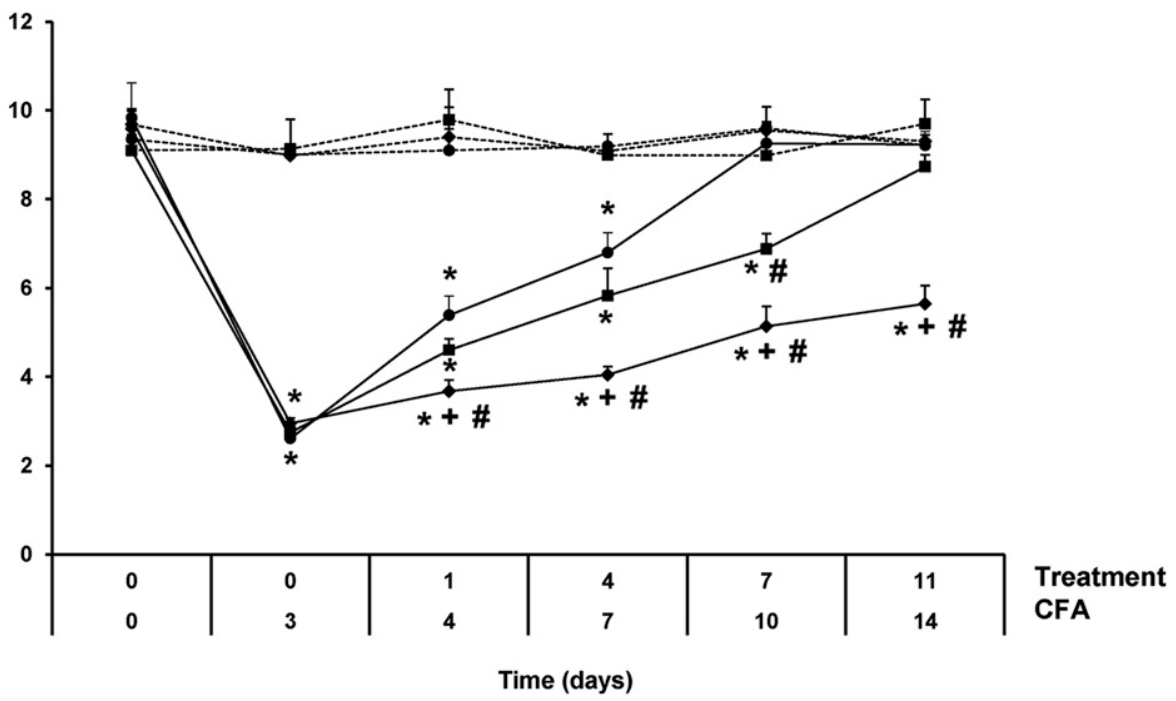

followed by the plantar test. After paw inflammation induction, animals were once again tested in each test at days 3, 4, 7, 10, and 14 after CFA injection as for baseline measures by using the same sequence. We used contralateral paws as controls ( $n=6$ animals per group). CFA-injected mice were administered 5 and $10 \mathrm{mg} / \mathrm{kg}$ of SFN, an Nrf2 transcription factor activator, or vehicle (dimethylsulfoxide $1 \%$ solution in saline) for 11 consecutive days from day 4 to 14 after CFA injection.

In a second set of studies, we evaluated the mechanical antiallodynic and thermal antihyperalgesic effects produced by $10 \mathrm{mg} / \mathrm{kg}$ of SFN intraperitoneally administered alone and in combination with a subanalgesic dose of morphine $(50 \mu \mathrm{g})$ subplantarly injected in the right hindpaw. Both contralateral and ipsilateral paws of CFAinjected animals were tested ( $n=6$ animals per group). The dose of SFN used for the combination was obtained from this study and the dose of morphine was selected from a previous study (Carcolé et al., 2014) as the one that displayed minimal antinociceptive effect. The researcher performing these experiments was blinded to the treatments applied.

The protein levels of Nrf2, HO-1, NQO1, NOS2, CD11b/c, MOR, and MAPK (p-JNK/total JNK, pERK1/2/total ERK1/2, and pp38/total p38) in the ipsilateral site of the spinal cord and paw from mice with peripheral inflammation and treated with SFN of vehicle were also
Fig. 1. Effects of repetitive treatment with SFN on the mechanical allodynia and thermal hyperalgesia induced by CFA. The development of the mechanical allodynia (A) and thermal hyperalgesia (B) in the ipsilateral paw (continuous lines) and contralateral paw (discontinuous lines) from mice treated for 11 consecTreatment utive days with vehicle or SFN at 5 or CFA $\quad 10 \mathrm{mg} / \mathrm{kg}$ are shown. Results of the evaluations at $3,4,7,10$, and 14 days after CFA injection are also shown. For each day and treatment evaluated, * indicates significant differences vs. their respective contralateral paw, + indicates significant differences vs. ipsilateral paws of animals treated with SFN at $5 \mathrm{mg} / \mathrm{kg}$, and \# indicates significant differences vs. ipsilateral paws of animals treated with SFN at $10 \mathrm{mg} / \mathrm{kg}(P<0.05$, one-way ANOVA followed by Student-NewmanKeuls test). Results are represented as mean \pm S.E.M. values; $n=6$ animals per experimental group. evaluated by western blot assay. Naive mice treated with vehicle were used as controls ( $n=4$ samples per group).

Drugs. While SFN was purchased from Merck Chemicals and Life Science S.A.U. (Madrid, Spain), morphine hydrochloride was acquired from Alcaiber S.A. (Madrid, Spain). SFN was dissolved in dimethylsulfoxide ( $1 \%$ in saline solution $0.9 \%$ ) and administered at 5 and $10 \mathrm{mg} / \mathrm{kg}$ intraperitoneally, in a final volume of $10 \mathrm{ml} / \mathrm{kg}$, at 3-4 hours prior to behavioral testing. Morphine hydrochloride was dissolved in saline solution $(0.9 \%)$ and subplantarly injected at $50 \mu \mathrm{g}$ into the right paw, in a final volume of $30 \mu \mathrm{l}$, at 30 minutes prior to behavioral testing. All drugs were prepared daily before administration. For each group treated with a drug, the respective control group received the same volume of the corresponding vehicle.

Statistical Analysis. Data are expressed as mean \pm S.E.M. The statistical analysis was accomplished by utilizing SPSS Statistics version 17 for Windows (IBM España, Madrid, Spain). In each behavioral test assessed, the effects of administration of 5 and $10 \mathrm{mg} / \mathrm{kg}$ of SFN versus the effects produced by the corresponding vehicle in the contralateral and ipsilateral paws of CFA-injected mice were compared using three-way analysis of variance (ANOVA) repeated measures (paw, treatment, and time as between factors of 


\section{IPSILATERAL}

A

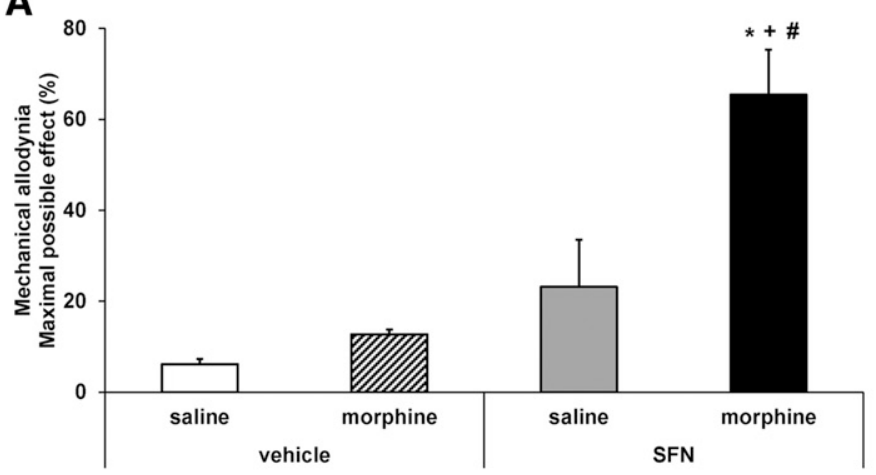

B

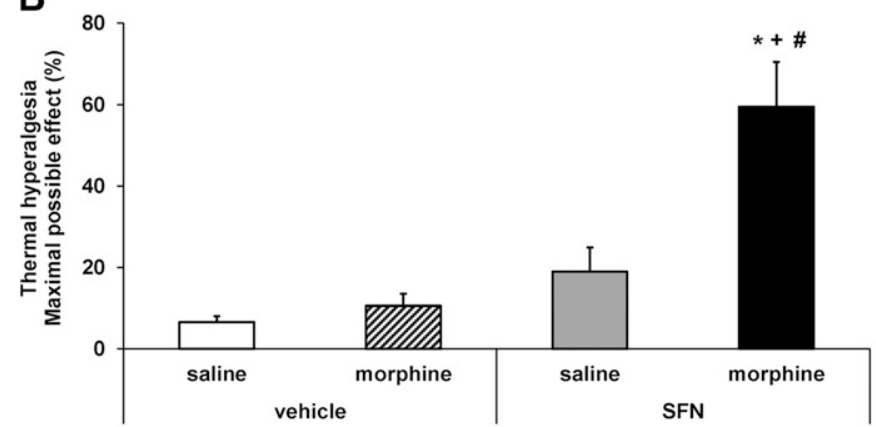

C

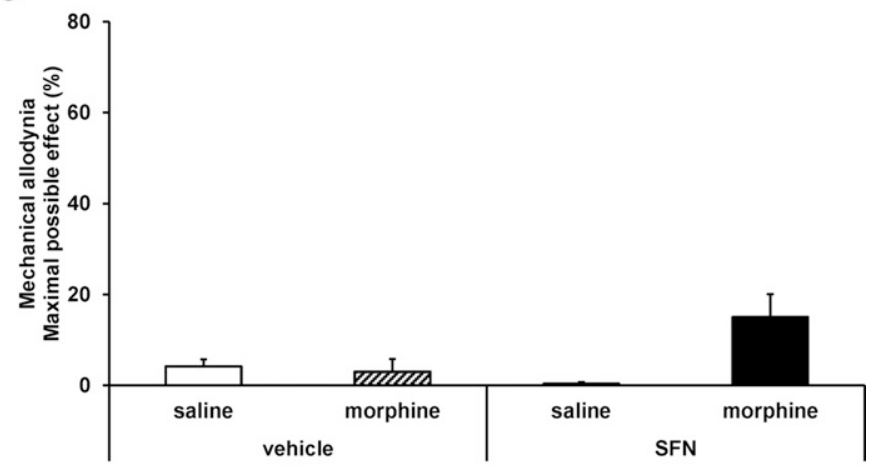

D

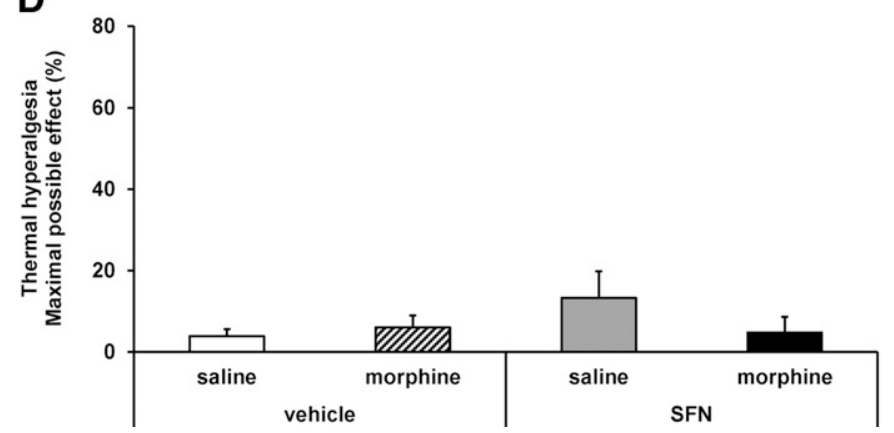

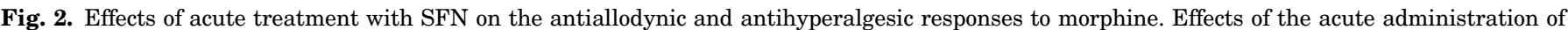

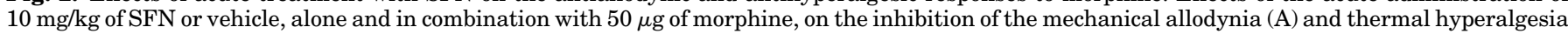

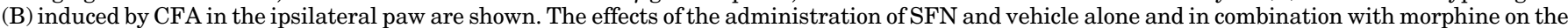

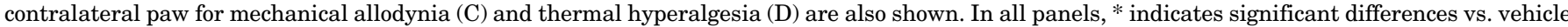

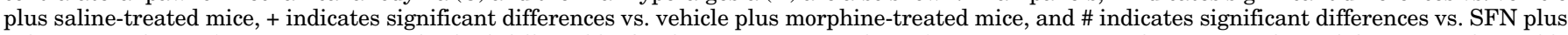

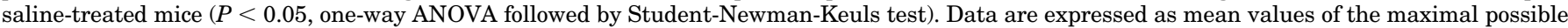
effect $(\%) \pm$ S.E.M.; $n=6$ animals per experimental group.

variation) followed by the appropriate one-way ANOVA and the Student-Newman-Keuls test whenever suitable.

In each behavioral test, the effects produced by SFN administered alone and in combination with morphine were compared using one-way ANOVA and the Student-Newman-Keuls test. In these studies, antinociception in the von Frey filaments and plantar tests is expressed as the percentage of maximal possible effect, where the test latencies predrug (baseline) and postdrug administration are compared and calculated in accordance with the following equation:

Maximal possible effect $(\%)=[($ drug - baseline $) /($ cut-off - baseline $)] \times 100$

Finally, changes in the protein levels of Nrf2, HO-1, NQO1, NOS2, $\mathrm{CD} 11 \mathrm{~b} / \mathrm{c}, \mathrm{MOR}$, and MAPK in the spinal cord and paw tissues from CFA-injected mice treated with SFN or vehicle and in naive mice receiving vehicle were examined using one-way ANOVA and the corresponding Student-Newman-Keuls test. $P<0.05$ was considered statistically significant.

\section{Results}

Effects of Repeated Treatment with SFN on the Mechanical Allodynia and Thermal Hyperalgesia Induced by CFA. Animals were intraperitoneally administered 5 and $10 \mathrm{mg} / \mathrm{kg}$ of SFN or vehicle for 11 consecutive days, starting the treatment at 4 days after CFA injection. At 1, 4, 7, and 11 days of SFN or vehicle treatment, mechanical allodynia and thermal hyperalgesia were sequentially assessed.

For mechanical allodynia, the subplantar administration of CFA significantly decreased the threshold for evoking hindpaw withdrawal to a mechanical stimulus. This effect was clearly observed after 3 days of CFA injection, and was maintained until day 14 in the ipsilateral paw of vehicletreated animals when compared with the respective contralateral paw ( $P<0.001$; one-way ANOVA) (Fig. 1A). The three-way ANOVA repeated measures test showed a significant effect of the paw, treatment, and time $(P<0.001)$ and the interactions between paw and treatment $(P<0.001)$, treatment and time $(P<0.001)$, paw and time $(P<0.001)$, and paw, treatment, and time $(P<0.001)$. Consequently, mechanical allodynia was significantly attenuated in mice daily treated with 5 and $10 \mathrm{mg} / \mathrm{kg}$ of SFN for 11 days $(P<0.001$, one-way ANOVA, compared with the ipsilateral paw of CFAinjected mice treated with vehicle) (Fig. 1A). However, while mechanical allodynia was equally reduced with the administration of 5 and $10 \mathrm{mg} / \mathrm{kg}$ of SFN on day 1 of treatment, the antiallodynic effects produced by $10 \mathrm{mg} / \mathrm{kg}$ of SFN after 7 days of treatment were higher than those obtained by the small dose ( $P<0.001$, one-way ANOVA). Moreover, although the antiallodynic effects produced by both doses increased progressively from days 1 to 7 of treatment $(P<0.001$, one-way ANOVA vs. the ipsilateral paw of CFA-injected mice treated 

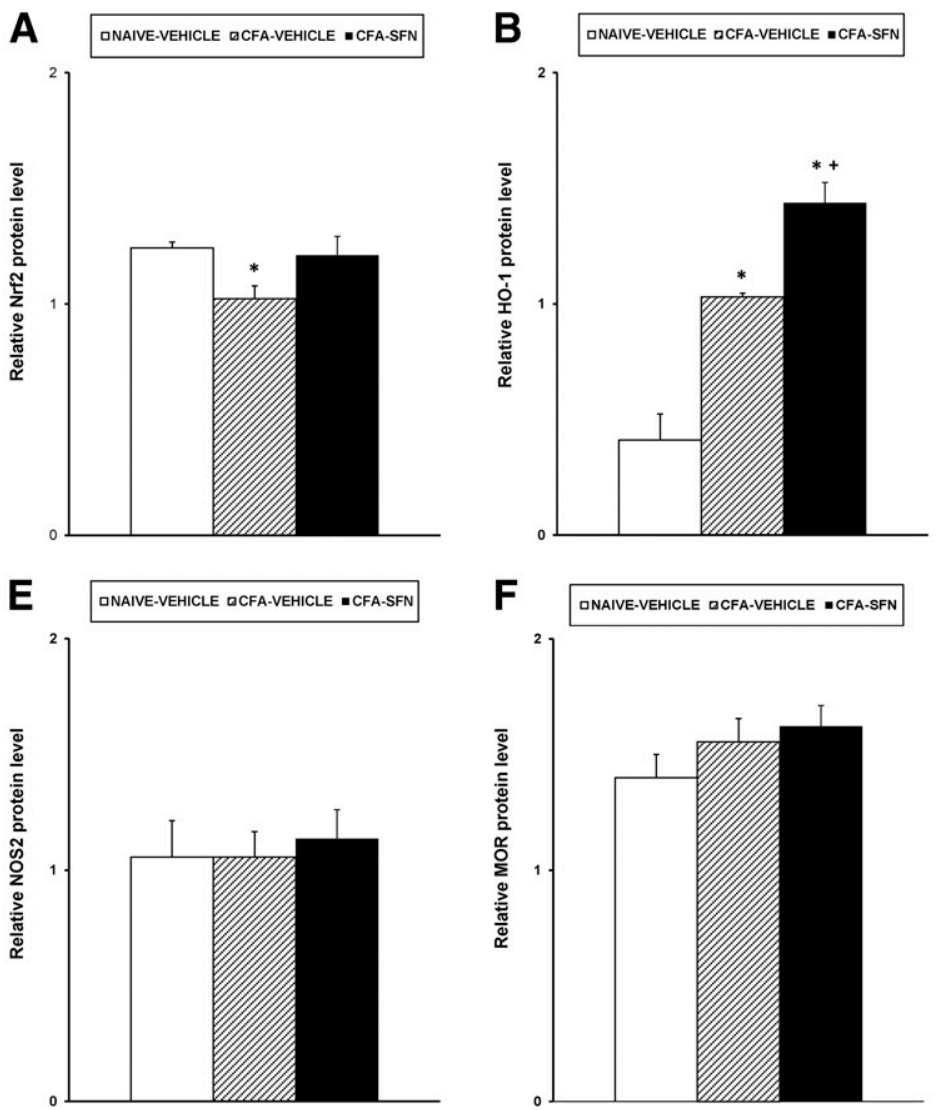

Fig. 3. Effects of treatment with SFN on the expression of Nrf2, HO-1, NQO1, NOS2, MOR, and CD11b/c in the spinal cord from animals with peripheral inflammation. The protein levels of Nrf2 (A), HO-1 (B), NQO1 (C), NOS2 (E), MOR (F), and CD11b/c (G) in the ipsilateral site of the spinal cord from CFAinjected mice treated with vehicle (CFA-VEHICLE) or SFN (CFA-SFN) are represented. The expression of these proteins in the spinal cord from naive mice treated with vehicle (NAIVE-VEHICLE) is also represented as controls. For each protein, * indicates significant differences when compared with naive vehicle-treated mice, + indicates significant differences when compared with CFA-injected mice treated with vehicle, and \# indicates significant differences when compared with CFA-injected mice treated with SFN $(P<0.05$, one-way ANOVA followed by Student-Newman-Keuls test). Representative examples of western blots are also shown for Nrf2, HO-1, and NQO1 proteins (D) and for NOS2, MOR, and CD11b/c (H), in which glyceraldehyde-3-phosphate dehydrogenase (GAPDH) was used as a loading control. Data are expressed as mean \pm S.E.M. values; $n=4$ samples per group.

with vehicle), mechanical allodynia was completely reversed at 7 days of treatment (10 days after CFA injection) with $10 \mathrm{mg} / \mathrm{kg}$ of SFN; however, 11 days of treatment with $5 \mathrm{mg} / \mathrm{kg}$ were needed to obtain this effect.

Peripheral inflammation also produced a significant decrease in the threshold for evoking ipsilateral paw withdrawal to a thermal stimulus in vehicle-treated mice from day 3 to day 14 after CFA injection compared with the corresponding contralateral paws ( $P<0.001$, one-way ANOVA) (Fig. 1B). The effects of intraperitoneal administration of SFN at 5 and $10 \mathrm{mg} / \mathrm{kg}$ were also evaluated. The three-way ANOVA repeated measures test showed a significant effect of the paw, treatment, and time $(P<0.001)$ as well as the interaction between paw and treatment $(P<0.001)$, treatment and time $(P<0.001)$, paw and time $(P<0.001)$, and paw, treatment, and time $(P<0.001)$ (Fig. 1B). Our results showed that thermal hyperalgesia caused by CFA was progressively attenuated after daily treatment with SFN (5 and $10 \mathrm{mg} / \mathrm{kg}$ ); similar to mechanical allodynia, complete reversion of thermal hyperalgesia was achieved at 7 and 11 days of treatment with 10 or $5 \mathrm{mg} / \mathrm{kg}$ of SFN, respectively $(P<0.001$, one-way
ANOVA, in comparison with the ipsilateral paw of mice with peripheral inflammation treated with vehicle).

Effects of Acute Treatment with SFN on the Antinociceptive Effects Produced by the Subplantar Administration of Morphine in Animals with Peripheral Inflammation. The effects of the acute intraperitoneal administration of $10 \mathrm{mg} / \mathrm{kg}$ SFN alone and in combination with $50 \mu \mathrm{g}$ morphine on the mechanical allodynia and thermal hyperalgesia caused by peripheral inflammation were evaluated. The data demonstrated that the intraperitoneal administration of SFN or morphine administered alone did not produce significant antiallodynic (Fig. 2A) and antihyperalgesic (Fig. 2B) effects compared with those produced by vehicle. SFN treatment significantly improved the mechanical antiallodynic (Fig. 2A) and thermal antihyperalgesic effects (Fig. 2B) produced by morphine locally administered in the ipsilateral paw of CFA-injected mice $(P<0.001$, one-way ANOVA vs. vehicle group treated with saline or morphine, or with SFN administered alone). The local administration of morphine alone or combined with SFN did not have any effect on the contralateral paw of CFA- 

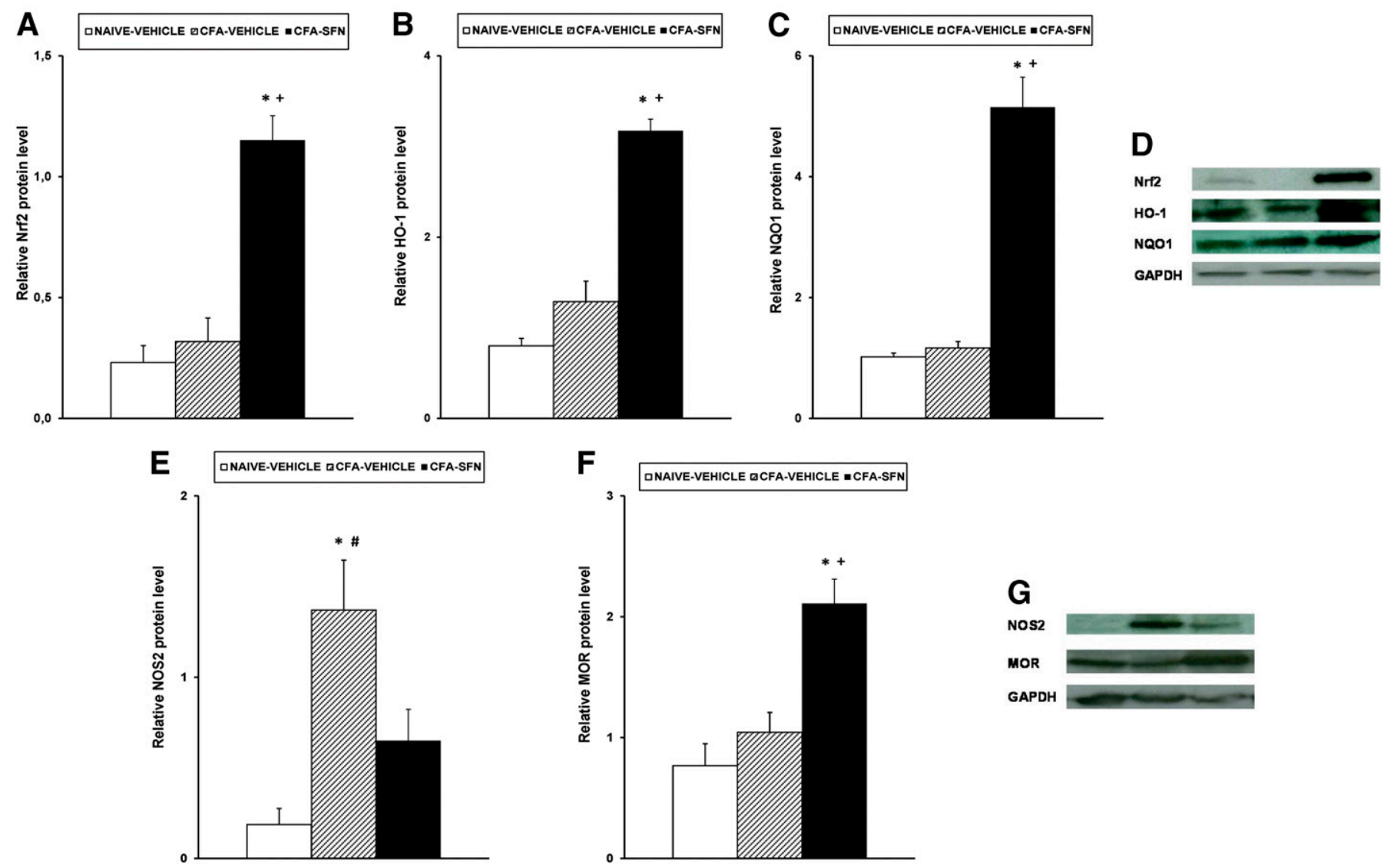

Fig. 4. Effects of treatment with SFN on the expression of Nrf2, HO-1, NQO1, NOS2, and MOR in the paw from animals with peripheral inflammation The protein levels of Nrf2 (A), HO-1 (B), NQO1 (C), NOS2 (E), and MOR (F) in the ipsilateral paw from CFA-injected mice treated with vehicle (CFAVEHICLE) or SFN (CFA-SFN) are represented. The expression of these proteins in the paw from naive mice treated with vehicle (NAIVE-VEHICLE) is also represented as controls. For each protein, * indicates significant differences when compared with naive vehicle-treated mice, + indicates significant differences when compared with CFA-injected mice treated with vehicle, and \# indicates significant differences when compared with CFA-injected mice treated with SFN $(P<0.05$, one-way ANOVA followed by Student-Newman-Keuls test). Representative examples of western blots are also shown for Nrf2, HO-1, and NQO1 proteins (D) and for NOS2 and MOR (G), in which glyceraldehyde-3-phosphate dehydrogenase (GAPDH) was used as a loading control. Data are expressed as mean \pm S.E.M. values; $n=4$ samples per group.

injected animals in relation to mechanical allodynia (Fig. 2C) and thermal hyperalgesia (Fig. 2D).

Effects of Repeated Treatment with SFN on the Nrf2, HO-1, NQO1, NOS2, MOR, and CD11b/c Expression in the Spinal Cord and Paw from Animals with Peripheral Inflammation. The Nrf2 levels and the expression of the antioxidant (HO-1 and NQO1) and inflammatory (NOS2) enzymes as well as of MOR and/or microglial marker (Cd11b/c) in the spinal cord (Fig. 3) and paw (Fig. 4) from CFA-injected mice treated for 11 days with $10 \mathrm{mg} / \mathrm{kg}$ of SFN or vehicle and from naive mice receiving vehicle were evaluated. Indeed, the diminished expression of Nrf2 in the spinal cord from CFAinjected mice $(P<0.01$, one-way ANOVA when compared with naive mice treated with vehicle) was normalized by treatment with SFN $(P<0.01$, one-way ANOVA vs. CFA-injected mice treated with vehicle) (Fig. 3A). A significant increased expression of Nrf2 induced by SFN was demonstrated in the paw from mice with peripheral inflammation $(P<0.01$, one-way ANOVA vs. naive and CFA-injected mice treated with vehicle) (Fig. 4A). Regarding HO-1, while peripheral inflammation increased the spinal cord levels of this enzyme $(P<0.001$, one-way ANOVA vs. naive vehicle treated mice) (Fig. 3B), nonstatistically significant alterations in its expression in the paw from CFA-injected mice was detected (Fig. 4B). Nevertheless, SFN treatment increased HO-1 protein levels in both tissues $(P<0.001$, one-way ANOVA vs. naive and CFAinjected mice treated with vehicle). Our findings also showed that SFN treatment enhanced the expression of NQO1 enzyme in the spinal cord (Fig. 3C) and paw (Fig. 4C) compared with naive and CFA-injected mice treated with vehicle $(P<0.01$, one-way ANOVA). Moreover, while peripheral inflammation did not modify the spinal cord levels of NOS2 (Fig. 3E), significant overexpression of this enzyme was observed in the paw $(P<0.004$, one-way ANOVA vs. naive mice treated with vehicle) (Fig. $4 \mathrm{E}$ ), which was significantly reversed by SFN administration. In addition, whereas the unaltered MOR levels observed in the spinal cord (Fig. 3F) and paw (Fig. 4F) from CFA-injected mice remained intact with SFN treatment in the spinal cord, a significant augmented expression of MOR was revealed in the paw tissues from SFNtreated animals $(P<0.028$ one-way ANOVA vs. naive and CFA-injected mice treated with vehicle). Finally, the spinal microglial activation caused by peripheral inflammation $(P<$ 0.002 one-way ANOVA vs. naive mice treated with vehicle) 


\section{SPINAL CORD}
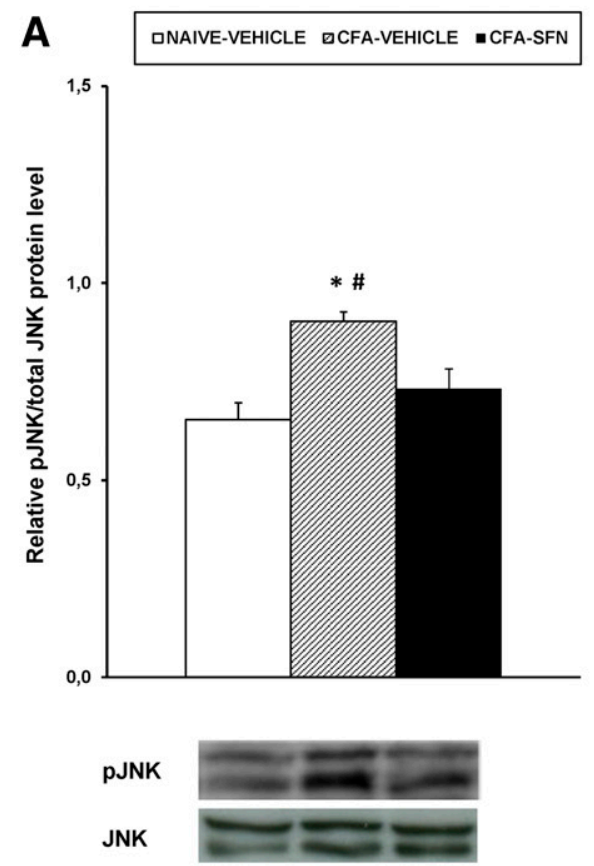

B

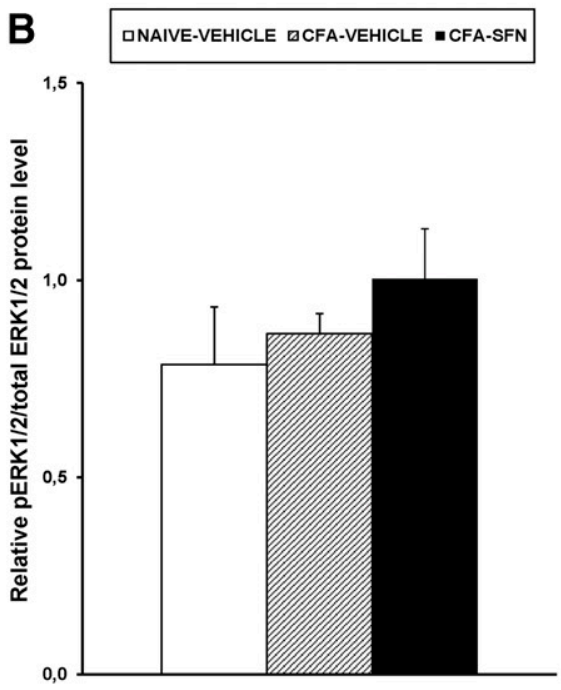

PERK $1 / 2$

ERK $1 / 2$
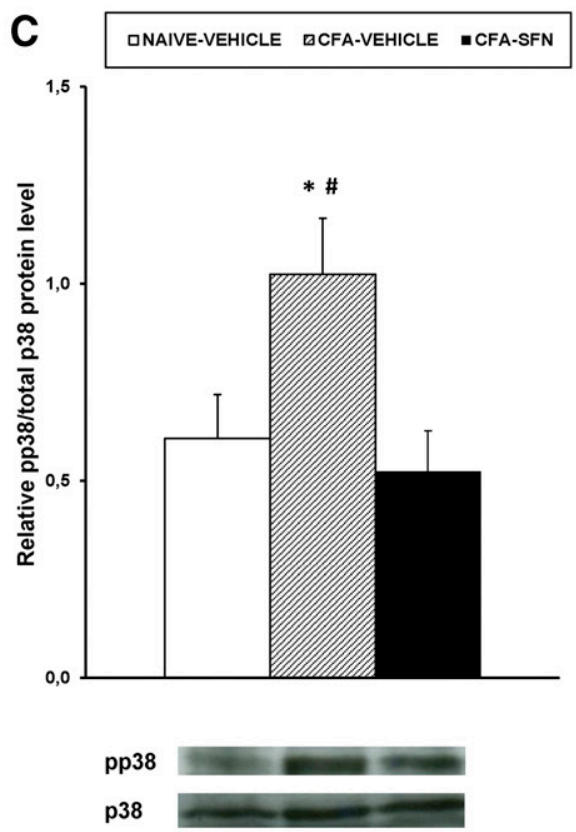

Fig. 5. Effects of treatment with SFN on the expression of pJNK, pERK1/2, and pp38 in the spinal cord from animals with peripheral inflammation. The protein levels of pJNK/total JNK (A), pERK1/2/total ERK1/2(B), and pp38/total p38 (C) in the ipsilateral site of the spinal cord from CFA-injected mice treated with vehicle (CFA-VEHICLE) or SFN (CFA-SFN) are represented. The expression of these proteins in the spinal cord from naive mice treated with vehicle (NAIVE-VEHICLE) is also represented as controls. For each protein, * indicates significant differences when compared with naive vehicletreated mice, and \# indicates significant differences when compared with CFA-injected mice treated with SFN $(P<0.05$, one-way ANOVA followed by Student-Newman-Keuls test). Representative examples of western blots are also shown for pJNK/total JNK (A), pERK1/2/total ERK1/2 (B), and pp38/total p38 (C) proteins. Data are expressed as mean \pm S.E.M. values; $n=4$ samples per group.

was totally reversed with $\mathrm{SFN}$ treatment $(P<0.002$ one-way ANOVA vs. CFA-injected mice treated with vehicle) (Fig. 3G).

Effects of Repeated Treatment with SFN on the Expression of JNK, ERK1/2, and p38 in the Spinal Cord and Paw from Animals with Peripheral Inflammation. The results revealed that the increased levels of phosphorylated JNK in the spinal cord (Fig. 5A) and paw (Fig. 6A) from CFA-injected mice $(P<0.05$ one-way ANOVA vs. naive mice treated with vehicle) were significantly diminished by SFN treatment in both tissues $(P<0.05$ one-way ANOVA vs. CFA-injected mice treated with vehicle). Regarding ERK1/2, while no changes in its expression were observed in the spinal cord (Fig. 5B), treatment with SFN prevented its activation in the paw from CFA-injected mice $(P<0.005$ oneway ANOVA vs. CFA-injected mice treated with vehicle) (Fig. 6B). Finally, the spinal cord activation of p38 induced by inflammation (Fig. 5C) was significantly reduced in SFNtreated mice $(P<0.028$ one-way ANOVA vs. CFA-injected mice treated with vehicle), while nonsignificant changes in the expression of pp38 were observed in the paw from CFA-injected mice (Fig. 6C).

\section{Discussion}

This study revealed that the repeated intraperitoneal administration of SFN, an activator of the transcription factor Nrf2, completely reversed the mechanical allodynia and thermal hyperalgesia caused by chronic peripheral inflammation in mice. This treatment activated the Nrf2/HO-1/NQO1 signaling, inhibited NOS2 overexpression and microglial activation, as well as MAPK phosphorylation in the spinal cord and/or paw tissues from CFA-injected animals. Our data also showed that SFN treatment improved the antiallodynic and antihyperalgesic effects produced by the subplantar injection of morphine by enhancing the local expression of MOR in animals with peripheral inflammation.

Several studies have demonstrated that the transcription factor Nrf2, which has antioxidant and neuroprotective effects, also plays an important role in the modulation of inflammation (Kobayashi et al., 2016). Therefore, loss or disruption of Nrf2 signaling causes increased susceptibility to oxidative stress and inflammatory tissue injuries (Kim et al., 2010). Other works have also shown the antinociceptive effects elicited by substances capable of activating the transcription factor Nrf2 during acute inflammatory pain (Rosa et al., 2008) and in neuropathic pain associated with type 1 and 2 diabetes (Negi et al., 2011; McDonnell et al., 2017). In this work we demonstrated that treatment with SFN (a Nrf2 activator) also decreased the mechanical and thermal hypersensitivity induced by chronic peripheral inflammation in a dose-dependent manner. That is, whereas 7 days of SFN treatment at $10 \mathrm{mg} / \mathrm{kg}$ are needed to inhibit inflammatory pain, 11 days of continuous administration of this drug at $5 \mathrm{mg} / \mathrm{kg}$ are required. These data support the preceding reports and furthermore demonstrate the antiallodynic and antihyperalgesic actions of SFN in chronic inflammatory pain in mice. 

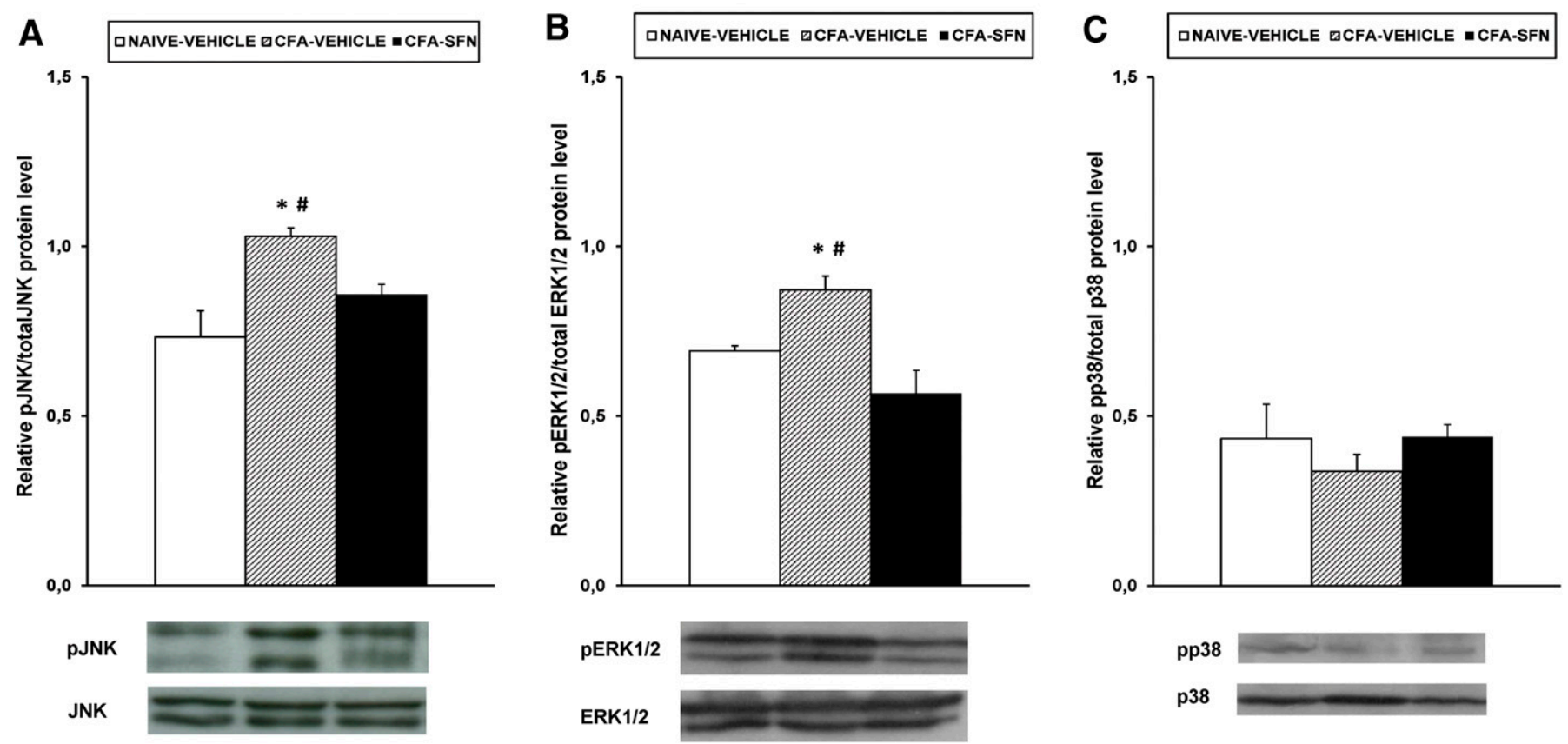

Fig. 6. Effects of treatment with SFN on the expression of pJNK, pERK1/2, and pp38 in the paw from animals with peripheral inflammation. The protein levels of pJNK/total JNK (A), pERK1/2/total ERK1/2 (B), and pp38/total p38 (C) in the ipsilateral paw from CFA-injected mice treated with vehicle (CFA-VEHICLE) or SFN (CFA-SFN) are represented. The expression of these proteins in the paw from naive mice treated with vehicle (NAIVEVEHICLE) is also represented as controls. For each protein, * indicates significant differences when compared with naive vehicle-treated mice and \# indicates significant differences when compared with CFA-injected mice treated with SFN $(P<0.05$, one-way ANOVA followed by Student-NewmanKeuls test). Representative examples of western blots are also shown for pJNK/total JNK (A), pERK1/2/total ERK1/2 (B), and pp38/total p38 (C) proteins. Data are expressed as mean \pm S.E.M. values; $n=4$ samples per group.

Our findings have also proven that administration of SFN avoids reduced levels of Nrf2 in the spinal cord and enhances its expression in the paw tissues from animals with inflammatory pain. This treatment also enhanced the downstream enzymes activated by Nrf2 (such as HO-1 and NQO1) in both tissues, suggesting that the antiallodynic and antihyperalgesic effects produced by SFN during inflammatory pain might be in part produced by its antioxidant activity mediated by the induction of these antioxidant enzymes. Supporting our results, the antinociceptive role played by the activation of HO-1 in inflammatory and neuropathic pain conditions have also been described (Hervera et al., 2012, 2013b; Carcolé et al., 2014). SFN treatment reduced the increased expression of NOS2 in the paw from CFA-injected mice. These data agree with the diminished NOS2 expression identified in the sciatic nerve from animals with diabetic neuropathy or nerve injury-induced neuropathic pain treated with SFN (Negi et al., 2011; Wang and Wang, 2017), as well as with HO-1 inducers (Castany et al., 2016), and further demonstrate the negative regulatory role played by Nrf2 in the expression of NOS2 under inflammatory pain conditions.

The involvement of microglia in the development of chronic pain is also well known. Using the expression of CD11b/c as a microglial marker, our data revealed significant microglial activation in the spinal cord of animals with peripheral inflammation, which was reversed by SFN treatment. Therefore, and considering the antinociceptive effects produced by specific microglial inhibitors in animals with chronic pain (Mika et al., 2009, 2013), the alleviation of inflammatory pain induced by SFN might, in part, have also been due to inhibiting spinal microglial activation.

To evaluate if the inhibition of MAPK activated by inflammatory pain is also involved in the effects produced by SFN, we investigated the expression of phosphorylated JNK, ERK1/2, and p38 in the spinal cord and paw tissues from mice treated with SFN. It is well known that the activation of JNK executes an important role in the development and maintenance of chronic pain (Middlemas et al., 2006; Gao and Ji, 2008). Our findings confirmed these results with the increased levels of phosphorylated JNK in the spinal cord as well as in the paw from mice with chronic inflammatory pain, and furthermore revealed that treatment with SFN completely reduced the JNK activation in both tissues. In agreement with our results, Gao et al. (2010) also demonstrated increased expression of JNK phosphorylated in the spinal cord from CFA-injected rats at 14 days after induction. Moreover, because treatments with specific JNK inhibitors reduced the mechanical allodynia caused by inflammation (Gao et al., 2010), the decreased activation of JNK performed by SFN might also contribute to explaining the antinociceptive effects produced by this Nrf2 inductor under inflammatory pain conditions. ERK1/2 is another member of MAPK, in which the phosphorylated form was also increased in response to several stimuli (inflammation, nerve injury, diabetes) at different levels of the pain processing pathway (Borges et al., 2015). Our data demonstrated that the augmented expression of pERK1/2 in the paw from mice with chronic inflammatory pain was 
significantly reduced by SFN treatment. In accordance with our findings, significant activation of pERK1/2 was also identified in the dorsal root ganglia from animals with acute and chronic peripheral inflammation (Cheng et al., 2008; Cheng and Keast, 2009; Gao and Ji, 2010). Since the administration of specific ERK1/2 inhibitors diminished the nociceptive response induced by inflammatory pain, the fact that SFN inhibited ERK phosphorylation in the paw also supports the hypothesis that the antinociceptive effects of this Nrf2 inductor during chronic inflammatory pain might also be mediated via inhibition of $\mathrm{pERK} 1 / 2$ in the paw. Finally, p38 activation is also responsible for the peripheral inflammation induced by hyperalgesia (Ji et al., 2002; Hudmon et al., 2008) and upregulation of numerous inflammatory mediators in the spinal cord (Svensson et al., 2003). Accordingly, significant increase in the levels of pp38 was detected in the spinal cord from CFA-injected mice that was completely blocked with the SFN treatment, revealing that the effectivity of this treatment against chronic inflammatory pain also includes modulation of p38 phosphorylation in the spinal cord of these animals. Finally, although we only assessed the effects of the systemic administration of SFN on the spinal cord and paw from animals with peripheral inflammation, it is probable that this treatment also produces relevant effects in other areas, such as the dorsal root ganglia, as occurs with other related treatments, for example, with the administration of HO-1 activators in mice with chronic pain (Hervera et al., 2012; Carcolé et al., 2014; Castany et al., 2016).

Our study demonstrated that the small antinociceptive effects produced by the local administration of a low dose of morphine during peripheral inflammation were significantly improved by SFN treatment. These results expanded our previous data indicating that the activation of HO-1 with cobalt protoporphyrin IX potentiated the peripheral antinociceptive actions of morphine in animals with inflammatory and neuropathic pain caused by nerve injury or associated with diabetes (Hervera et al., 2013b; Carcolé et al., 2014; Castany et al., 2016) and further revealed that the activation of Nrf2 might also potentiate the analgesic effects of morphine under inflammatory pain conditions. Moreover, the enhancement of MOR expression induced by SFN in the paw might explain the improvement of the local antiallodynic and antihyperalgesic effects of morphine elicited by SFN in animals with inflammatory pain. Due to our results indicating that SFN induced the synthesis of $\mathrm{HO}-1$, which also upregulated the expression of MOR in the dorsal root ganglia from CFA-injected mice (Carcolé et al., 2014), we hypothesized that the activation of the Nrf2/HO-1 signaling pathway might be the main effect responsible for the enhanced expression of MOR observed in the paw from SFN-treated animals. Nonetheless, the antioxidant and anti-inflammatory effects triggered by SFN administration might also contribute to the improvement of the local antinociceptive effects of morphine produced by this treatment.

In summary, this study indicated that the induction of Nrf2 might inhibit inflammatory pain and enhance the analgesic effects of morphine by inhibiting oxidative stress and inflammatory responses caused by peripheral inflammation. This study suggests the administration of SFN alone and in combination with morphine are potential new ways of treating chronic inflammatory pain.

\section{Authorship Contributions}

Participated in research design: Pol.

Conducted experiments: Redondo, Ferreira Chamorro, Riego, Leánez. Performed data analysis: Redondo, Pol.

Wrote or contributed to the writing of the manuscript: Pol.

\section{References}

Borges G, Berrocoso E, Mico JA, and Neto F (2015) ERK1/2: function, signaling and implication in pain and pain-related anxio-depressive disorders. Prog Neuropsychopharmacol Biol Psychiatry 60:77-92.

Carcolé M, Castany S, Leánez S, and Pol O (2014) Treatment with a heme oxygenase 1 inducer enhances the antinociceptive effects of $\mu$-opioid, $\delta$-opioid, and cannabinoid 2 receptors during inflammatory pain. J Pharmacol Exp Ther 351:224-232.

Castany S, Carcolé M, Leánez S, and Pol O (2016) The induction of heme oxygenase 1 decreases painful diabetic neuropathy and enhances the antinociceptive effects of morphine in diabetic mice. PLoS One 11:e0146427.

Chaplan SR, Bach FW, Pogrel JW, Chung JM, and Yaksh TL (1994) Quantitative assessment of tactile allodynia in the rat paw. $J$ Neurosci Methods 53:55-63.

Cheng HT, Suzuki M, Hegarty DM, Xu Q, Weyerbacher AR, South SM, Ohata M, and Inturrisi CE (2008) Inflammatory pain-induced signaling events following a conditional deletion of the N-methyl-D-aspartate receptor in spinal cord dorsal horn. Neuroscience 155:948-958.

Cheng Y and Keast JR (2009) Effects of estrogens and bladder inflammation on mitogen-activated protein kinases in lumbosacral dorsal root ganglia from adult female rats. BMC Neurosci 10:156.

Davidson RK, Jupp O, de Ferrars R, Kay CD, Culley KL, Norton R, Driscoll C, Vincent TL, Donell ST, Bao Y, et al. (2013) Sulforaphane represses matrixdegrading proteases and protects cartilage from destruction in vitro and in vivo. Arthritis Rheum 65:3130-3140.

Di W, Shi X, Lv H, Liu J, Zhang H, Li Z, and Fang Y (2016) Activation of the nuclear factor E2-related factor 2/anitioxidant response element alleviates the nitroglycerin-induced hyperalgesia in rats. $J$ Headache Pain 17:99.

Gao YJ and Ji RR (2008) Activation of JNK pathway in persistent pain. Neurosci Lett 437:180-183.

Gao YJ and Ji RR (2010) Light touch induces ERK activation in superficial dorsal horn neurons after inflammation: involvement of spinal astrocytes and JNK signaling in touch-evoked central sensitization and mechanical allodynia. J Neurochem 115:505-514.

Gao YJ, Xu ZZ, Liu YC, Wen YR, Decosterd I, and Ji RR (2010) The c-Jun N-terminal kinase 1 (JNK1) in spinal astrocytes is required for the maintenance of bilateral mechanical allodynia under a persistent inflammatory pain condition. Pain 148: 309-319.

Hargreaves K, Dubner R, Brown F, Flores C, and Joris J (1988) A new and sensitive method for measuring thermal nociception in cutaneous hyperalgesia. Pain $\mathbf{3 2}$ 77-88.

Hervera A, Gou G, Leánez S, and Pol O (2013a) Effects of treatment with a carbon monoxide-releasing molecule and a heme oxygenase 1 inducer in the antinociceptive effects of morphine in different models of acute and chronic pain in mice. Psychopharmacology (Berl) 228:463-477.

Hervera A, Leánez S, Motterlini R, and Pol O (2013b) Treatment with carbon monoxidereleasing molecules and an HO-1 inducer enhances the effects and expression of $\mu$-opioid receptors during neuropathic pain. Anesthesiology 118:1180-1197.

Hervera A, Leánez S, Negrete R, Motterlini R, and Pol O (2012) Carbon monoxide reduces neuropathic pain and spinal microglial activation by inhibiting nitric oxide synthesis in mice. PLoS One 7:e43693.

Hervera A, Leánez S, Negrete R, and Pol O (2009) The peripheral administration of a nitric oxide donor potentiates the local antinociceptive effects of a DOR agonist during chronic inflammatory pain in mice. Naunyn Schmiedebergs Arch Pharmacol 380:345-352.

Hudmon A, Choi JS, Tyrrell L, Black JA, Rush AM, Waxman SG, and Dib-Hajj SD (2008) Phosphorylation of sodium channel $\mathrm{Na}_{\mathrm{v}} 1.8$ by p38 mitogen-activated protein kinase increases current density in dorsal root ganglion neurons. J Neurosci 28:3190-3201.

Ji RR, Gereau RW, IV, Malcangio M, and Strichartz GR (2009) MAP kinase and pain. Brain Res Brain Res Rev 60:135-148.

Ji RR, Samad TA, Jin SX, Schmoll R, and Woolf CJ (2002) p38 MAPK activation by NGF in primary sensory neurons after inflammation increases TRPV1 levels and maintains heat hyperalgesia. Neuron 36:57-68.

Kim HA, Yeo Y, Kim WU, and Kim S (2009) Phase 2 enzyme inducer sulphoraphane blocks matrix metalloproteinase production in articular chondrocytes. Rheumatology (Oxford) 48:932-938

Kim J, Cha YN, and Surh YJ (2010) A protective role of nuclear factor-erythroid 2-related factor-2 (Nrf2) in inflammatory disorders. Mutat Res 690:12-23.

Kobayashi EH, Suzuki T, Funayama R, Nagashima T, Hayashi M, Sekine H, Tanaka N, Moriguchi T, Motohashi H, Nakayama K, et al. (2016) Nrf2 suppresses macrophage inflammatory response by blocking proinflammatory cytokine transcription. Nat Commun 7:11624

Leánez S, Hervera A, and Pol O (2009) Peripheral antinociceptive effects of $\mu$ - and $\delta$-opioid receptor agonists in NOS2 and NOS1 knockout mice during chronic inflammatory pain. Eur J Pharmacol 602:41-49.

Maicas N, Ferrándiz ML, Brines R, Ibáñez L, Cuadrado A, Koenders MI, van den Berg WB, and Alcaraz MJ (2011) Deficiency of Nrf2 accelerates the effector phase of arthritis and aggravates joint disease. Antioxid Redox Signal 15:889-901.

Martini AC, Berta T, Forner S, Chen G, Bento AF, Ji RR, and Rae GA (2016) Lipoxin A4 inhibits microglial activation and reduces neuroinflammation and neuropathic pain after spinal cord hemisection. J Neuroinflammation 13:75.

McDonnell C, Leánez S, and Pol O (2017) The induction of the transcription factor Nrf2 enhances the antinociceptive effects of delta-opioid receptors in diabetic mice. PLoS One 12:e0180998. 
Middlemas AB, Agthong S, and Tomlinson DR (2006) Phosphorylation of c-Jun N-terminal kinase (JNK) in sensory neurones of diabetic rats, with possible effects on nerve conduction and neuropathic pain: prevention with an aldose reductase inhibitor. Diabetologia 49:580-587.

Mika J, Osikowicz M, Rojewska E, Korostynski M, Wawrzczak-Bargiela A, Przewlocki R, and Przewlocka B (2009) Differential activation of spinal microglial and astroglial cells in a mouse model of peripheral neuropathic pain. Eur $J$ Pharmacol 623:65-72.

Mika J, Zychowska M, Popiolek-Barczyk K, Rojewska E, and Przewlocka B (2013) Importance of glial activation in neuropathic pain. Eur $J$ Pharmacol 716:106-119.

Negi G, Kumar A, and Sharma SS (2011) Nrf2 and NF- $\mathrm{B}$ B modulation by sulforaphane counteracts multiple manifestations of diabetic neuropathy in rats and high glucose-induced changes. Curr Neurovasc Res 8:294-304.

Pol O (2007) The involvement of the nitric oxide in the effects and expression of opioid receptors during peripheral inflammation. Curr Med Chem 14:1945-1955.
Rosa AO, Egea J, Lorrio S, Rojo AI, Cuadrado A, and López MG (2008) Nrf2-mediated haeme oxygenase-1 up-regulation induced by cobalt protoporphyrin has antinociceptive effects against inflammatory pain in the formalin test in mice. Pain 137:332-339.

Svensson CI, Hua XY, Protter AA, Powell HC, and Yaksh TL (2003) Spinal p38 MAP kinase is necessary for NMDA-induced spinal PGE(2) release and thermal hyperalgesia. Neuroreport 14:1153-1157.

Wang C and Wang C (2017) Anti-nociceptive and anti-inflammatory actions of sulforaphane in chronic constriction injury-induced neuropathic pain mice. Inflammopharmacology 25:99-106.

Address correspondence to: Dr. Olga Pol, Grup de Neurofarmacologia Molecular, Institut d'Investigació Biomèdica Sant Pau \& Institut de Neurociències, Facultat de Medicina. Edifici M2-115. Universitat Autònoma de Barcelona, 08193 Bellaterra, Barcelona, Spain. E-mail: opol@santpau.es 